\title{
Identification of Two Distinct Muscles in the Planarian Dugesia japonica by their Expression of Myosin Heavy Chain Genes
}

\author{
Chiyoko Kobayashi, Seitaro Kobayashi, Hidefumi Orii, \\ Kenji Watanabe and Kiyokazu Agata* \\ Laboratory of Regeneration Biology, Department of Life Science, Faculty of Science, \\ Himeji Institute of Technology, Hyogo 678-1297, Japan
}

\begin{abstract}
Ultrastructural and physiological studies have shown that planarian muscles have some characteristics of smooth and some characteristics of striated muscles. To characterize planarian muscles, we isolated two myosin heavy chain genes (DjMHC-A and DjMHC-B) from a planarian, Dugesia japonica, by immunological screening, and analyzed their structures and spatial expression patterns. Structural analysis indicated that both MHC genes are striated-muscle-type myosin genes, although planarian muscles do not have any striation. In situ RNA hybridization showed that expression of the two myosin genes is spatially strictly segregated. DjMHC-A was expressed in pharynx muscles, pharynx cavity muscles, muscles surrounding the intestinal ducts, a subpopulation of body-wall muscles and several muscle cells in the mesenchymal region around the base of the pharynx. DjMHC-B was expressed in body-wall muscles (including circular, diagonal and longitudinal muscles), vertical muscles and horizontally oriented muscles. Double staining with $D j M H C-A$ and $-B$ probes clearly demonstrated that expression of the DjMHC-A and - $B$ genes do not occur in the same cell. During regeneration, the number of cells positive for expression of each gene increased in the blastema region, suggesting that both types of muscle may be involved in blastema formation. DjMHC-B-positive cells disappeared from the body-wall muscle layer in the pharynx-cavity-forming region, whereas $D j M H C$-A-positive cells were markedly accumulated there, suggesting that the two types of muscle in the body wall layer may have distinct functions. These results indicate that planarians have at least two types of muscle that express striated-muscle-type MHC genes, but do not form striation.
\end{abstract}

\section{INTRODUCTION}

The muscles of most invertebrates and vertebrates can be clearly classified as striated or smooth muscles by their morphological characteristics. However, oblique muscles, which occur in some invertebrates, have characteristics intermediate between those of striated and smooth muscles (Hanson, 1956; Lowy and Hanson, 1962), i.e., they contain dense bodies characteristic of smooth muscles although they have helical striation composed of myofilaments. Muscles of planarians have even more ambiguous characteristics (MacRae, 1963; Morita, 1965; Sarnat, 1984). Their structure resembles that of smooth muscle, since they have no striation and contain dense bodies, but their nuclei are located in peripheral regions of the cells, which is characteristic of striated muscles, and a Z-line-like structure directly connected to sarcolemma is also observed (Hori, 1983). We have therefore begun to clarify the nature of planarian muscles by using cloned genes to probe the expression of muscle-specific genes.

It is well known that many organisms have multiple isoforms of myosin heavy chains (MHCs), which are classified into three types by their structure and expression pattern: striated muscle, smooth muscle and nonmuscle types (Warrick and Spudich, 1987). Thus, it is expected that MHC genes should be good landmark genes to classify morphologically ambiguous planarian muscles at the molecular level. A short fragment of an $\mathrm{MHC}$ gene of a planarian, Girardra tigrina, has already been cloned by immunological screening using a muscle-specific monoclonal antibody (Bueno et al., 1997; Cebria et al., 1997). However, only one MHC gene has been cloned so far using that method, and the cloned cDNA fragment is too short to fully characterize the MHC gene(s) of planarians. Here we report the structure and expression patterns of two different MHC genes isolated from the planarian Dugesia japonica, and describe some resultant insights into the characteristics and evolution of planarian muscles.

\footnotetext{
* Corresponding author: Tel. +81-7915-8-0187; FAX. +81-7915-8-0187.
} 


\section{MATERIALS AND METHODS}

\section{Animals}

A clonal strain of planarian, Dugesia japonica, established in our laboratory, was used. It was bred in boiled tap water at $22-24^{\circ} \mathrm{C}$, and fed chicken liver twice a week. All worms were used for experiments after one week of starvation.

\section{Cloning and DNA sequencing}

Mouse antiserum against living planarians was used to screen a $\lambda Z A P$ expression cDNA library of planarian. Immunological screening was performed according to the manufacture protocols (Stratagene). Forty-seven positive clones among $4 \times 10^{4} \mathrm{cDNA}$ clones were classified by cross-reactivity and restriction enzyme analyses. These positive clones were recloned into pBluescript by in vivo excision. Purified plasmid DNA was sequenced using a BcaBEST Dideoxy Sequencing Kit (Takara) or a Thermo Sequence Fluorescent Labeled Primer Cycle Sequencing Kit (Amersham). Analysis of the sequence was performed using the DDBJ on-line service (National Institute of Genetics, Japan) or the Wisconsin GCG Package program (Version 9.0, Genetics Computer Group, Madison, WI, USA).

\section{In situ hybridization of sections and whole-mount specimens}

Planarians were treated with $2 \% \mathrm{HCl}$ for $5 \mathrm{~min}$ and fixed either in relaxant solution $\left(1 \% \mathrm{HNO}_{3}, 1.6 \%\right.$ formaldehyde, $20 \mu \mathrm{M} \mathrm{MgSO}_{4}$; Dawar, 1973) for $12-24 \mathrm{hr}$, or in $2 \%$ formaldehyde, $0.2 \%$ saturated picric acid solution for $30 \mathrm{~min}$, followed by $2 \%$ formaldehyde, $0.2 \%$ saturated picric acid solution, $0.1 \%$ glutaraldehyde for $1 \mathrm{hr}$, and then $2 \%$ formaldehyde, $0.2 \%$ saturated picric acid solution for $2 \mathrm{hr}$. They were then dehydrated through a graded ethanol series, embedded in paraffin, sectioned horizontally, sagittally or transversely and then mounted on poly-L-lysine-coated slides. After de-paraffination in xylene and hydration through the ethanol series, the mounted specimens were rinsed in PBS and acetylated in $0.1 \mathrm{M}$ triethanolamine, $0.09 \% \mathrm{HCl}$ and $0.25 \%$ acetic anhydride for $15 \mathrm{~min}$. They were rinsed in $2 \times \mathrm{SSC}$ and then incubated at $55^{\circ} \mathrm{C}$ for $30 \mathrm{~min}$ in $50 \%$ formamide, $5 \times \mathrm{SSC}$ and $10 \mathrm{mM}$ DTT. Digoxygenin-labeled antisense probe derived from $D j M H C$ - $A$ or $D j M H C-B$ was diluted to $50 \mathrm{ng}-500 \mathrm{ng} / \mathrm{ml}$ in $50 \%$ formamide, $5 \times \mathrm{SSC}, 0.1 \mathrm{mg} / \mathrm{ml}$ yeast RNA, $0.1 \mathrm{mg} / \mathrm{ml}$ heparin, $0.1 \%$ Tween-20, $10 \mathrm{mM}$ DTT and $10 \%$ dextran sulfate, denatured at $65^{\circ} \mathrm{C}$ for $15 \mathrm{~min}$, and added to slides for hybridization at $55^{\circ} \mathrm{C}$ for 16 $36 \mathrm{hr}$. After hybridization, the slides were washed in $50 \%$ formamide, $5 \times \mathrm{SSC}$ and $0.1 \%$ Tween-20 for $9-12 \mathrm{hr}$ at $55^{\circ} \mathrm{C}$, and twice in $2 \times$ $\mathrm{SSC}$ at room temperature for $15 \mathrm{~min}$. The slides were rinsed in Buffer$1(0.1 \mathrm{M}$ maleic acid, $0.15 \mathrm{M} \mathrm{NaCl}, 0.1 \%$ TritonX-100), incubated in Buffer-2 (1\% Boehringer blocking reagent in Buffer-1) for 30 min and then in Buffer-2 containing alkaline-phosphatase (AP)-conjugated antidigoxygenin antibody (Boehringer) diluted 1:5000 at room temperature for $2 \mathrm{hr}$. They were rinsed in Buffer-1 and in TMN buffer $(0.1 \mathrm{M}$ $\mathrm{NaCl}, 0.1 \mathrm{M}$ Tris- $\mathrm{HCl}, \mathrm{pH}$ 9.5, 0.05 $\mathrm{M} \mathrm{MgCl}_{2}$ ) for $15 \mathrm{~min}$. Signals were rendered detectable by incubation of the slides in TMN buffer containing $200 \mu \mathrm{g} / \mathrm{ml} \mathrm{NBT} \mathrm{(Sigma)} \mathrm{and} 175 \mu \mathrm{g} / \mathrm{ml} \mathrm{BCIP} \mathrm{(Sigma)} \mathrm{for} \mathrm{2-3}$ $\mathrm{hr}$. After the chromogenic reaction was completed, the slides were washed twice in TE buffer (10 mM Tris- $\mathrm{HCl}, \mathrm{pH} 8,1 \mathrm{mM}$ EDTA), mounted in $50 \%$ glycerol in TE buffer and observed under a microscope. Double staining was performed using digoxigenin-labeled probe for MHC-B and biotin-labeled probe for MHC-A. Both signals were simultaneously detected using a TSA Kit (NEN Life Science Products) according to the procedures of Kato et al. (manuscript in preparation). Whole-mount in situ hybridization was carried out as described by Umesono et al. (1997) and Agata et al. (1998).

\section{Dot blot hybridization}

Non-labeled sense RNAs synthesized from DjMHC-A or DjMHC$B$ and yeast tRNA were diluted serially and blotted on nylon membranes (Hybond-N, Amersham) in duplicate. The RNA was fixed to the membrane by baking at $80^{\circ} \mathrm{C}$ for $2 \mathrm{hr}$ and cross-linking by UV light. Both membranes were incubated in prehybridization solution at $55^{\circ} \mathrm{C}$ for $1 \mathrm{hr}$. They were then incubated with the probe at $50 \mathrm{ng} / \mathrm{ml}$ at $55^{\circ} \mathrm{C}$ for $16-36 \mathrm{hr}$. After hybridization, they were washed in the wash buffer at $55^{\circ} \mathrm{C}$ for $9-12 \mathrm{hr}$ and washed twice in $2 \times \mathrm{SSC}$ at room temperature for $15 \mathrm{~min}$. The hybridization signal was detected immunologically as described above.

\section{RESULTS}

Isolation and characterization of two planarian MHC genes

Forty-seven clones that reacted with anti-planarian antiserum were classified into two groups (A and $B$ ) by crossreactivity and restriction enzyme analyses. The longest clone of group A contained a 6111-bp insert with an ORF of 1958 amino acids. The longest clone of group B contained a 5388bp insert with an ORF of 1743 amino acids. The nucleotide sequence data reported in this paper will appear in the DDBJ/ EMBL/GenBank nucleotide sequence databases with the accession numbers AB015484 and AB015485.

Computer analysis of the DNA and amino acid sequences showed high similarity between the two genes $(79.9 \%$ at the protein level) and similarity of both genes to myosin heavy chain genes of other animals (Fig. 1). The predicted amino acid sequence of the group-A gene shows $64.4 \%$ similarity to that of scallop and $53.1 \%$ similarity to that of human MHC. That of the group-B gene shows $64.2 \%$ and $52.8 \%$ similarity to those of scallop and human MHCs, respectively. Myosinspecific domains such as the ATP-binding site, actin-binding sites, two closely spaced reactive thiol groups; $\mathrm{SH} 1$ and $\mathrm{SH} 2$, and hinge were well conserved. Therefore, we named the group-A and -B genes "DjMHC-A" and "DjMHC-B", respectively.

To classify these two planarian MHCs, we compared them to striated-muscle-type and smooth-muscle-type MHCs of other organisms. Figure 2A shows a comparison of the amino acid sequences of the isoform-specific region among $D j M H C$ $A, D j M H C-B$ and MHCs of various other organisms (Schuchert et al., 1993; Takahashi et al., 1992). This comparison indicated that both $D j M H C-A$ and $D j M H C-B$ genes are striatedmuscle-type MHC genes since they showed much higher similarities to striated-muscle-type than to smooth- or nonmuscletype MHCs. Phylogenetic tree analysis also suggested that both planarian MHC genes should be classified as striatedmuscle type. DjMHC- $A$ and $D j M H C-B$ are related more closely to each other than to any other MHC gene. Although these structural analyses suggest that both MHCs are striatedmuscle-type MHCs, no striation has been observed in planarian muscles (MacRae, 1963; Morita, 1965; Hori, 1983).

\section{Comparison between DjMHC-A and DjMHC-B}

We next compared the structures of DjMHC-A and $D j M H C-B$. The overall identities between DjMHC-A and DjMHC- $B$ are $74.9 \%$ and $79.9 \%$ at the DNA and protein levels, respectively. HarrProt analysis clearly indicated regions conserved between DjMHC-A and DjMHC-B (Fig. 3A). The head region is highly homologous between them (92.2\%), but the light meromyosin (LMM) region of the tail is much less 


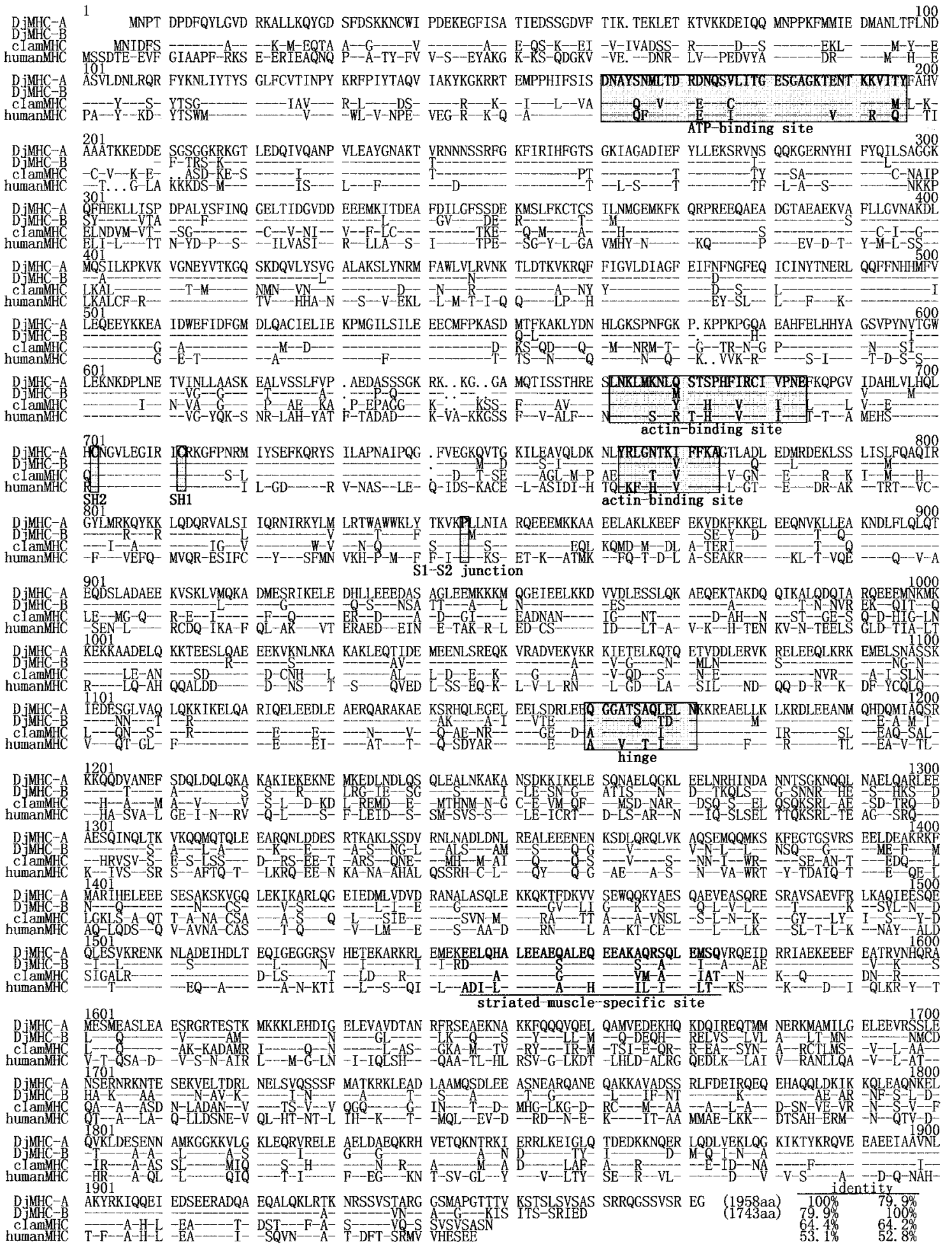

Fig. 1. Predicted amino acid sequences of $D j M H C-A$ and $D j M H C-B$ compared to those of clam (scallop) and human MHCs. Conserved amino acids are indicated by dashes. Dots indicate gaps for alignment. Myosin-specific domains of the MHCs are boxed. Underlines indicate amino acid sequences conserved among the striated-muscle MHCs.

homologous $(70.4 \%)$. Since the two genes show strong homology in the head region, we detected cross-reaction between them during routine hybridization procedures. Thus, we needed to establish conditions for avoiding cross-reaction during in situ hybridization. First, we used tail regions as probes, since these regions have low homology. However, the resultant hybridization signals were weaker than the signals obtained using full-length probes (data not shown). There- 
A

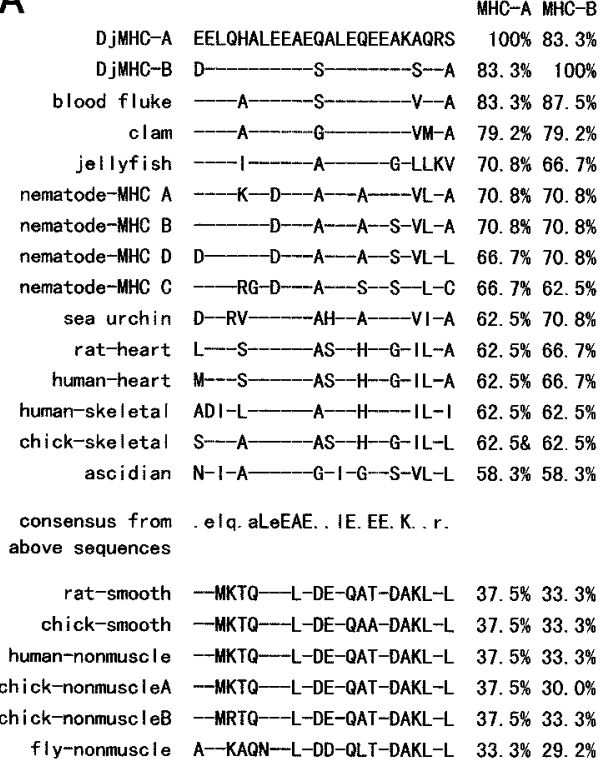

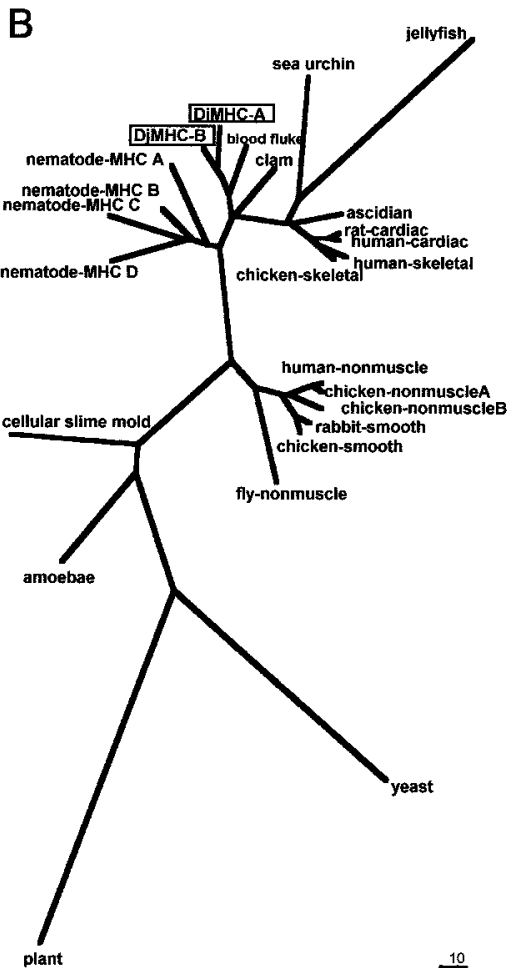

Fig. 2. Comparison of amino acid sequences in the isoform-specific region, and phylogenic tree analysis. (A) Alignment of the sequences in the isoform-specific region (Schuchert et al., 1993). Complete sequences were aligned by the pileup program of the GCG Package and then the isoform-specific region was selected. Sequences were taken from the SWISS Plot and DDBJ/GenBank/EMBL sequence libraries. Dashes indicate identical residues. The consensus sequence of the striated-muscle-type MHCs is indicated. Capital letters indicate perfect conservation; lower case letters, high conservation; points, no conservation. (B) An unrooted phylogenetic tree of DjMHC-A, DjMHC-B and other MHCs. This tree was produced using the distances program of the GCG Package. The accession numbers and amino acid residues of the MHCs used in these analyses are as follows: amoebae, P05659; ascidian, D45163; blood fluke, L01634; cellular slime mold, P08799; chicken-skeletal, P13538; chicken-nonmuscleA, P14105; chicken-nonmuscleB, M93676, PID g212449; chicken-smooth, P10587; clam, P24733; fly-nonmuscle, Q99323; human-nonmuscle, P35579; human-skeletal, P11055; human-cardiac, P12883; jellyfish, X64057; nematode-MHC A, P12844; nematode-MHC B, P02566; nematode-MHC C, P12845; nematode-MHC D, P02567; plant, Z34293; rabbit-smooth, P35748; rat-cardiac, P02563; sea urchin, M55321; yeast, P08964. Accession numbers that begin with a "P" are from the SWISS Plot database; all others are from the DDBJ/GenBank/ EMBL database. "10" indicates the phylogenetic distance expressed as substitutions per 100 amino acids.

A

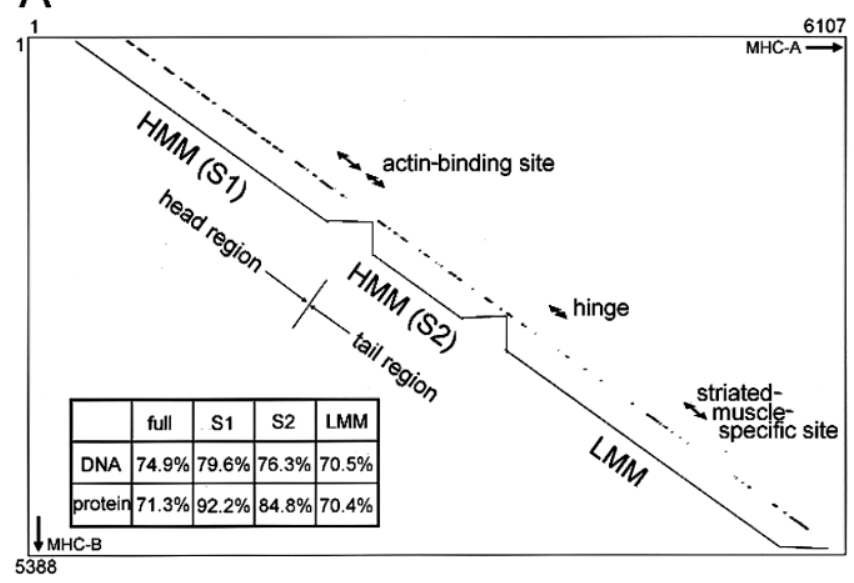

B

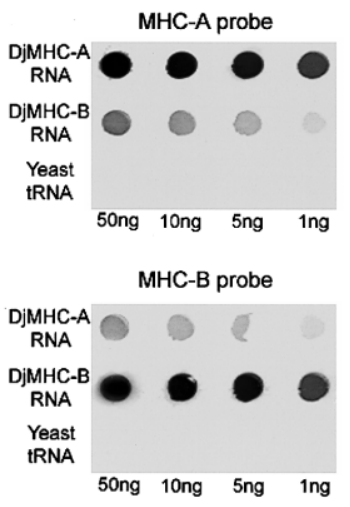

Fig. 3. Comparison between structures of $D j M H C-A$ and $D j M H C-B$. (A) HarrProt analysis was performed to compare the $D j M H C-A$ and $D j M H C$ $B$ genes. Unit size used for the comparison: 30, Dot plot matching number: 25. HMM, heavy meromyosin; LMM, light meromyosin; S1, subunit 1; $\mathrm{S} 2$, subunit 2. The table summarizes the identity between $D j M H C$ - $A$ and $D j M H C-B$ in three regions. (B) Cross-reactivity between $D j M H C-A$ and $D j M H C-B$ in high stringency condition. The sense RNAs of DjMHC-A and DjMHC-B, and yeast tRNA were diluted serially and blotted on nylon membranes. Dot blot hybridization was performed using labeled antisense RNA probes: DjMHC-A (upper panel), DjMHC-B (lower panel). 
fore, we tried performing RNA hybridization at $55^{\circ} \mathrm{C}$ in a solution containing $50 \%$ formamide, and checked the cross-reactivity between $D j M H C-A$ and $D j M H C-B$ by serial dilution experiments (Fig. 3B). We obtained satisfactory results using these high stringency conditions, although slight cross-reactivity was detected.

\section{Expression of DjMHC-A and DjMHC-B in normal adult pla- narians}

To investigate the spatial distribution of planarian $\mathrm{MHC}$ gene transcripts in normal adult planarians, we performed in situ hybridization under the high stringency conditions described above. Strong expression of $D j M H C$-A was detected in pharynx muscles (Fig. 4A-a, b and d). DjMHC-A-positive signals were also observed in muscles surrounding the intestinal ducts (Fig. 4A-c and d; arrows), a subpopulation of bodywall muscles (Fig. 4A-c; arrowheads), several cells in the mesenchymal region around the base of the pharynx (Fig. 4A-C; asterisks), and pharynx-cavity muscles (Fig. 4A-d; arrowheads).

In contrast to the pattern seen with $D j M H C$ - $A$, expression of DjMHC-B was detected in body-wall muscles (Fig. 4Ba, b and c; arrows), vertical muscles (Fig. 4B-a and b; arrowheads) and horizontally oriented muscles in the ventral part of the body (Fig. 4B-c; short arrows), but not in pharynx muscles. Interestingly, we found that the nucleus of the vertical muscles is located in the dorsal region (Fig. 4B-C; open arrowheads), whereas the nucleus of the horizontally oriented muscles is located in the ventral region (Fig. 4B-c; arrowheads).

Since we detected expression of both genes in the bodywall-muscle layer and the mesenchymal region, we performed doubly probed in situ hybridizations to investigate whether or not $D j M H C$ - $A$ and $D j M H C$ - $B$ were expressed in the same cells. In Fig. 5, DjMHC-A- and DjMHC-B-positive cells are shown in green and red colors, respectively. Blue color indicates nuclear counter-staining using Hoechst 33342. No overlapping expression was observed. In the mesenchymal region, DjMHC-Apositive cells were distributed at the periphery of the intestinal duct, but $D j M H C$ - $B$-positive cells were observed in the space between the intestinal ducts (Fig. 5B-D). Figure 5E-G shows higher magnification views of the body-wall layer. DjMHC-A and $D j M H C$ - $B$ expression was observed in cells in the same layer, but not in the same cells. These results clearly demonstrate that muscle cells of planarians can be classified into at least two types by their patterns of expression of $\mathrm{MHC}$ genes.

\section{Expression of $D j M H C$ - $A$ and $D j M H C$ - $B$ in regenerating pla- narians}

We analyzed the expression patterns of the two $\mathrm{MHC}$ genes during regeneration. Intact planarians were cut behind the pharynx, and the resultant regenerating planarians were analyzed by in situ hybridization. During regeneration, the cells expressing each MHC gene in the body-wall-muscle layer increased specifically in the blastema region (Fig. $6 \mathrm{~A}$ and $\mathrm{B}$; the blastema region is surrounded by dots). Furthermore, $D j M H C-B$ expressing cells were also increased in the region inside of the blastema region. Interesting views were obtained during the process of pharynx-cavity formation. After 5-6 days of regeneration, the pharynx cavity becomes open. Around that time, DjMHC-B-positive cells disappeared from the bodywall-muscle layer in the nascent cavity-forming region (Fig. $6 \mathrm{D}$, asterisk), whereas $D j M H C$ - $A$-positive cells were accumulated there (Fig. 6C, asterisk). Such complementary expression patterns suggest that DjMHC-A- and DjMHC-B-expressing cells may have distinct functions in the body-wall-muscle layer.

\section{DISCUSSION}

We have isolated two MHC genes, DjMHC-A and DjMHC$B$, from a planarian, Dugesia japonica, and analyzed their structures and expression patterns. Surprisingly, both the $D j M H C-A$ and $D j M H C-B$ genes were of the striated-muscle type, although no striation was observed in planarian muscles (MacRae, 1963; Morita, 1965; Hori, 1983). We expected that the muscles surrounding the intestinal ducts might express an $\mathrm{MHC}$ gene of the smooth-muscle type, as in vertebrates. However, we were not able to isolate smooth-muscle-type $\mathrm{MHC}$ genes from planarian. The muscles surrounding the intestinal ducts express a striated-muscle-type MHC gene, $D j M H C-A$. In the case of $C$. elegans, four MHC genes have been isolated and all of them are classified as striated-muscle type: the MHC-A and -B genes are expressed in body-wall muscles, while the MHC-C and -D genes are expressed in pharynx muscles (Dibb et al., 1988; Epistein et al., 1974; Garcea et al., 1978; Miller et al., 1986: Fig. 2B). Morphological studies indicate that all the muscles of C.elegans are oblique muscles (Burr and Gans, 1998). These results obtained in planarians and nematodes suggest that all the muscle myosins of invertebrates may be encoded by striated-muscle-type $\mathrm{MHC}$ genes. Smooth muscles in vertebrates may have evolved from cells in other lineages, based on nonmuscle-type MHC genes.

Analysis of expression of DjMHC-A and DjMHC-B clearly indicated that planarian muscles are composed of at least two distinct types of muscles. DjMHC-A is specifically expressed in pharynx muscles, muscles surrounding the intestinal ducts, pharynx-cavity muscles and a subpopulation of body-wall muscles (Fig. 7A and B; green). On the other hand, DjMHC-B is expressed in body-wall muscles, vertical muscles and horizontally oriented muscles (Fig. 7A and B; red). These expression patterns suggest that the $D j M H C$ - $A$-expressing muscles may have a function related to peristaltic movement, and that the $D j M H C$ - $B$-expressing muscles may have a function related to body movement. These functional differences may be important in pharynx-cavity formation during regeneration. DjMHC-B-expressing muscles disappeared completely from the body-wall layer in the cavity-forming region, whereas $D j M H C$ - $A$-expressing muscles were accumulated there.

To understand the evolution of muscles, it is also interesting to analyze composition and expression pattern of actin genes in planarians. Since it has been well documented 

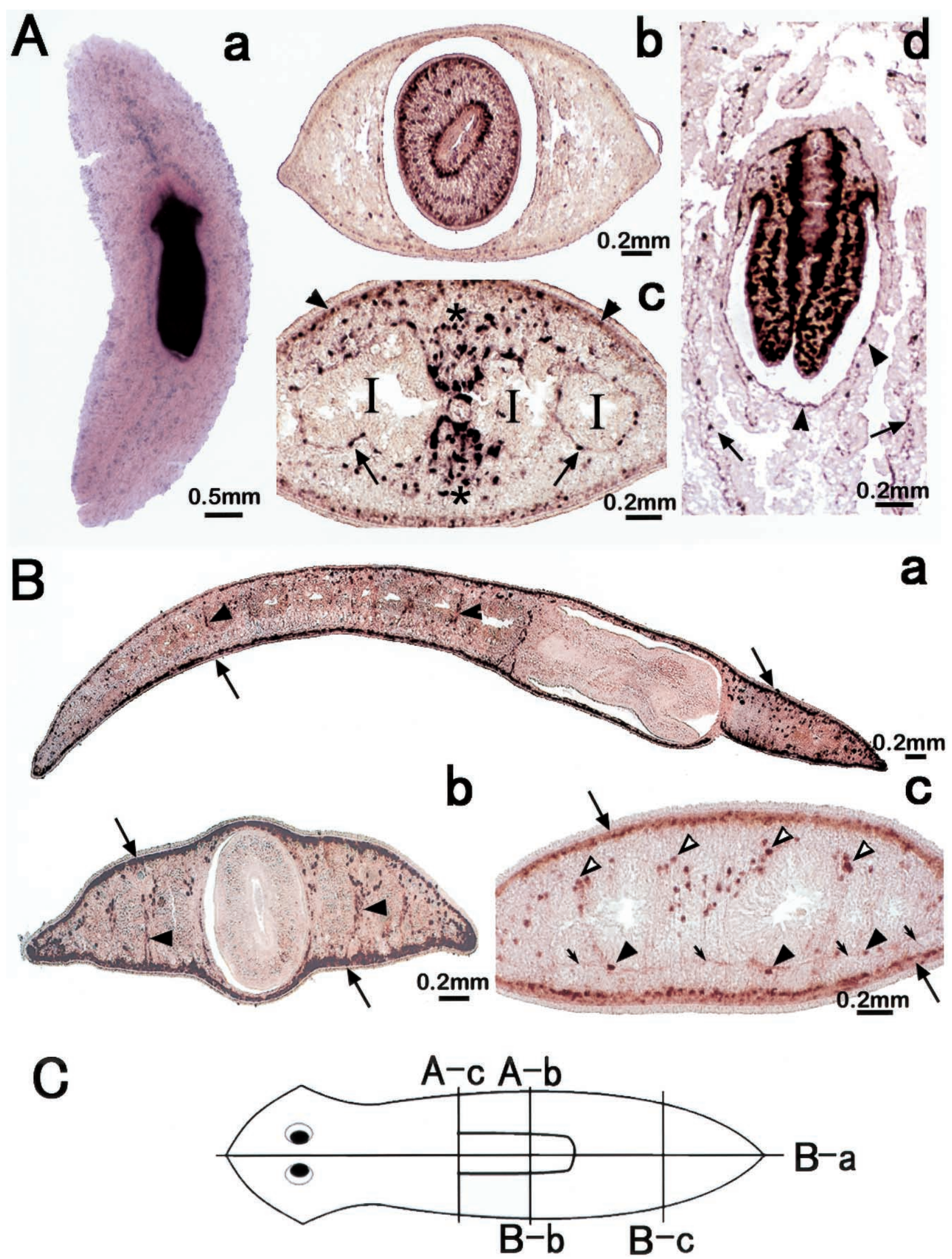
A
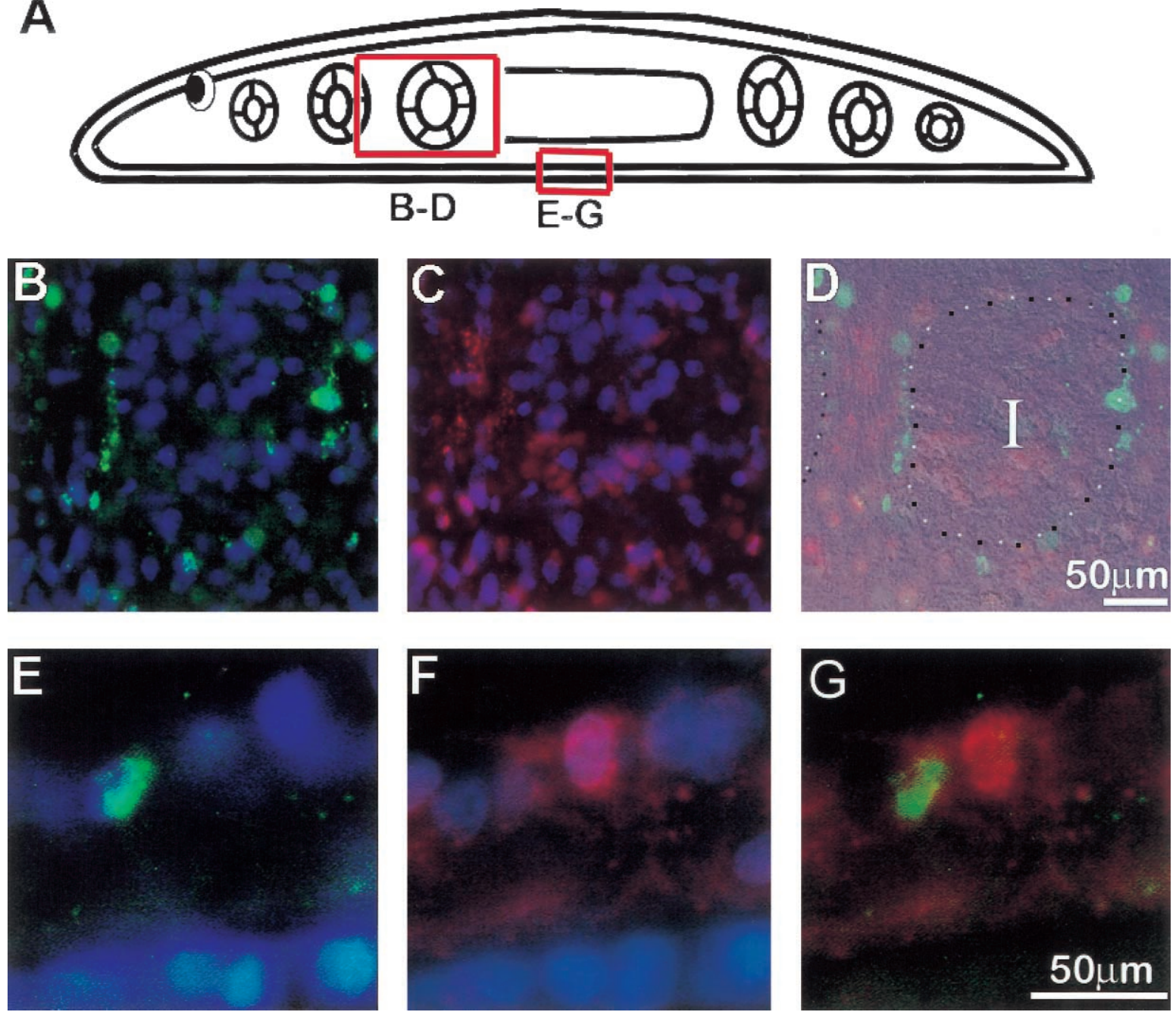

Fig. 5. Doubly stained in situ hybridization of sagittal sections using the DjMHC-A and DjMHC-B probes. Nuclei were counter-stained with Hoechst 33342 (blue). (A) A diagram of the position of sections. (B-D) Intestine and transverse muscle area. (B) Expression of $D j M H C-A$ was observed in the muscles surrounding the intestinal duct (green). (C) Expression of DjMHC-B was observed in transverse muscles (red). (D) A double exposure of figures (B) and (C); DjMHC-A (green) and DjMHC-B (red). Dots indicate the intestinae. (E-G) Body-wall area. (E) Expression of $D j M H C-A$ (green) (F) Expression of $D j M H C-B$ (red). (G) A double exposure of figures (E) and (F); $D j M H C-A$ (green) and $D j M H C-B($ red). I, intestinal duct. Scale bars, $50 \mu \mathrm{m}$.

Fig. 4. Expression patterns of $D j M H C-A$ and $D j M H C-B$ in normal adult planarians. (A) Expression of DjMHC-A. (a) A whole-mount in situ hybridization. (b) A transverse section of the pharynx region. (c) A transverse section of the base of the pharynx. Arrowheads indicate expression in body-wall muscles. Arrows indicate expression in the muscles surrounding the intestinal ducts. Asterisks indicate expression in the cells in the mesenchymal space. (d) A high-magnification view of the pharynx region in a horizontal section. Arrows indicate expression in the pharynx-cavity muscle. Arrows indicate expression in the muscles surrounding the intestinal ducts. (B) Expression of DjMHC-B. (a-c) Arrows indicate expression in body-wall muscles. (a) A sagittal section. Arrowheads indicate transverse muscles. (b) A transverse section of the pharynx region. Arrowheads indicate transverse muscles. (c) A transverse section of the tail region. Short Arrows indicate horizontally oriented muscles. Open arrowheads indicate nucleus of the transverse muscles. Arrows indicate nucleus of the horizontally oriented muscles. (C) A diagram of the position of sections. I, intestinal duct. Scale bars, $0.5 \mathrm{~mm}(\mathrm{Aa})$ and $0.2 \mathrm{~mm}$ (Ab$\mathrm{d}$ and $\mathrm{Ba}-\mathrm{c})$. that the vertebrates have isoform-specific actin genes expressed in tissue- or developmental stage-specific manner (Vandekerckhove and Weber, 1979). Recently, such tissueor developmental stage-specific expression patterns of actin genes of the invertebrates were also indicated in ascidians and arrow worms (Kusakabe et al., 1991; Kusakabe et al., 1995; Yasuda et al., 1997). We need to describe expression pattern of actin genes in planarians and compare it with that of MHC genes to understand the characteristics and evolution of planarian muscles.

Cells expressing the DjMHC- $A$ gene were observed in the mesenchymal region around the base of the pharynx, and they appear to participate in pharynx formation (Fig. 4A-c; asterisks). If they are pharynx-forming muscles, pharynx muscles may differentiate in the mesenchymal space and then migrate into the pharynx. We will need to clarify whether or not these 

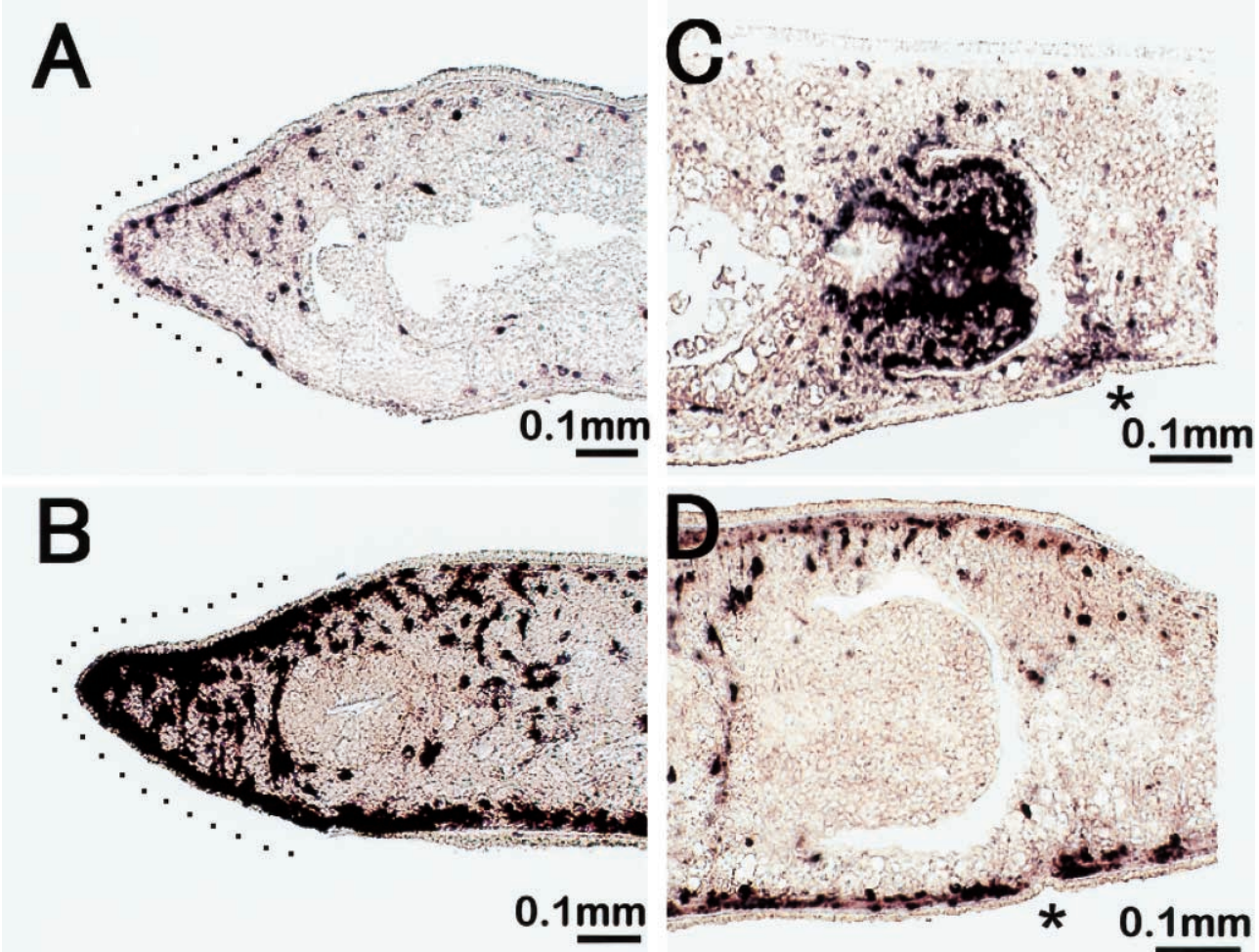

Fig. 6. Expression patterns of $D j M H C-A$ and $D j M H C-B$ during the process of regeneration from tail piece. (A, B) Sagittal sections 2 days after amputation. The blastema region is surrounded by dots. (A) Expression of $D j M H C$ - $A$. (B) Expression of $D j M H C$ - $B$. (C, D) Sagittal sections 5 days after amputation. Asterisks indicate the pharynx-cavity-forming region. (C) Expression of DjMHC-A. (D) Expression of $D j M H C-B$. Scale bars, $0.1 \mathrm{~mm}$.
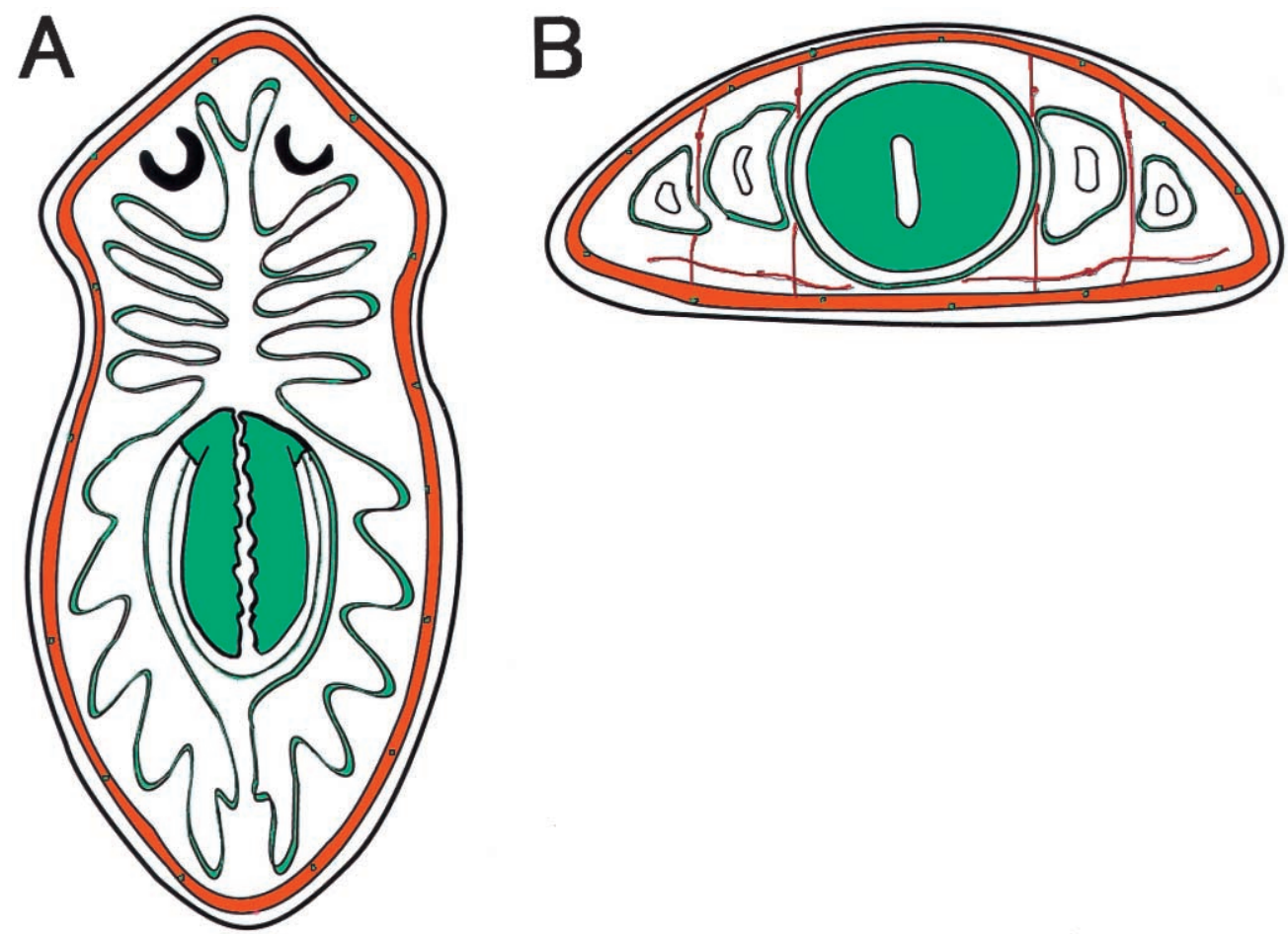

Fig. 7. Summary of the expression domains of $D j M H C-A$ (green) and $D j M H C-B$ (red). (A) A horizontal section. (B) A transverse section. 
cells are pharynx-forming muscles, in order to understand the mechanism of pharynx regeneration in planarians.

Unexpectedly, we could not isolate genes other than the two MHC genes by the $\lambda$ ZAP expression-cloning system in spite of using the antiserum against living planarians. All of the forty-seven positive clones contained cDNA fragments more than $5.7 \mathrm{~kb}$ in size. Surprisingly, sequence analyses revealed that most of the cDNA fragments had been fused to the $L a c Z$ gene out of frame. These results suggested that the $\mathrm{MHC}$ products may be translated from internal methionines in the 5 ' regions. When we changed the expression-cloning system from the $\lambda$ ZAP system to the $\lambda$ gt 11 system, we succeeded in isolating a variety of genes with the same antiserum (data not shown). We therefore concluded that the characteristics of the $\lambda$ ZAP system may be the reason why MHC genes were selectively isolated by immunoscreening using the antiserum against living planarians as a probe.

\section{ACKNOWLEDGMENTS}

We thank Dr. Y. Umesono for encouragement. This work was supported by Special Coordination Funds for Promoting Science and Technology to K.A. and Grant-in-Aid for Scientific Research on Priority Areas to K.A. and K.W.

\section{REFERENCES}

Agata K, Soejima Y, Kato K, Kobayashi C, Umesono Y, Watanabe K (1998) Structure of the planarian central nervous system (CNS) revealed by neuronal cell markers. Zool Sci 15: 433-440

Bueno D, Baguñà J, Romeo R (1997) Cell-, tissue-, and positionspecific monoclonal antibodies against the planarian Dugesia (Girardia) tigrina. Histochem Cell Biol 107: 139-149

Burr AH, Gans C (1998) Mechanical significance of obliquely striated architecture in nematode muscle. Biol Bull 194: 1-6

Cebria F, Vispo M, Newmark P, Bueno D, Romero R (1997) Myocyte differentiation and body wall muscle regeneration in the planarian Girardia tigrina. Dev Genes Evol 207: 306-316

Dibb NJ, Maruyama IN, Krause M, Karn J (1988) Sequence analysis of the Complete Caenorhabditis elegans myosin heavy chain gene family. J Mol Biol 205: 603-613

Dowar BL (1973) A combined relaxing agent and fixative for triclads (planarians). Stain Technol 48: 96-94

Epstein HF, Waterston RH, Brenner S (1974) A mutant affecting the heavy chain of myosin Caenorhabditis elegans. J Mol Biol 90: 291-300
Garcea RL, Schachat F, Epstain HF (1978) Coordinate synthesis of two myosins in wild-type and mutant nematode muscle during larval development. Cell 15: 421-428

Hanson J (1956) The structure of the smooth muscle fibres in the body wall of the earthworm. J Biophysic Biochem Cytol 3: 111-127

Hori I (1983) Differentiation of myoblasts in the regeneationg planarian Dugesia japonica. Cell Differ 12: 155-163

Kusakabe T, Suzuki J, Saiga H, Jeffery WR, Makabe KW, Satoh N (1991) Temporal and spatial expression of a muscle actin gene during embryogenesis of the ascidean Halocynthia roretzi. Dev Growth Differ 33: 227-234

Kusakabe T, Hikosaka A, Satoh N (1995) Coexpression and promoter function in two muscle actin gene complexes of different structural organization in the ascidean Halocynthis roretzi. Dev Biol 169: 461-472

Lowy J, Hanson J (1962) Ultrastructure of invertebrate smooth muscle. Physiol Rev 42, Suppl 5: 34-47

MacRae EK (1963) Observation on the fine structure of pharyngeal muscle in the planarian Dugesia tigrina. J Cell Biol 18:651-662

Miller DM, Stockdale FE, Kern J (1986) Immunological identification of the genes encoding the four myosin heavy chain isoforms of Caenorhabditis elegans. Proc Nat Acad Sci USA 83: 2305-2309

Morita M (1965) Electron microscopic studies on planaria. I. Fine structure of muscle fiber in the head of the planarian Dugesia dorotocephala. J Ultrastruct Res 13: 383-395

Sarnat HB (1984) Muscle histochemistry of the planarian Dugesia tigrina (Turbellaria: Tricladida): implications in the evolution of muscle. Trans Am Microsc Soc 103: 284-294

Schuchert P, Reber-Müller S, Schmid V (1993) Life stage specific expression of a myosin heavy chain in the hydrozoan Podocoryne carnea. Differentiation 51: 11-18

Takahashi M, Kawamoto S, Adelstein RS (1992) Evidence for inserted sequences in the head region of non-muscle myosin specific to the nervous system. J Biol Chem 267: 17864-17871

Umesono Y, Watanabe K, Agata K (1997) A planarian orthopedia homolog is specifically expressed in the branch region of both the mature and regenerating brain. Dev Growth Differ 39: 723-727

Vandekerckhove J, Weber K (1979) The complete amino acid sequence of actins from bovine aorta, bovine heart, bovine fast skeletal muscle and rabbit slow skeletal muscle. Differentiation 14: 123-133

Warrick HM, Spudich JA (1987) Myosin structure and function in cell motility. Ann Rev Cell Biol 3: 379-421

Yasuda E, Goto T, Makabe KW, Satoh N (1997) Expression of actin genes in the arrow worm Paraspadella gotoi (Chaetognatha). Zool Sci 14: 953-960

(Received June 30, 1998 / Accepted August 6, 1998) 